УДК 532.61.096

\title{
Equilibrium and Stability of a Free Liquid Film in a Longitudinal Gravitational Field
}

\author{
Oksana A. Burmistrova* \\ Lavrentyev Institute of Hydrodynamics of SB RAS \\ Lavrentyev prospect, 15, Novosibirsk, 630090 \\ Russia
}

Received 24.03.2015, received in revised form 30.04.2015, accepted 06.05.2015

\begin{abstract}
Deformation of the free vertical liquid film by combined effect of gravity and thermocapillary forces is investigated. The system of equations connecting the flow rate, the film thickness and its temperature is found in the framework of thin-layer approximation. Using the shooting method, one-dimensional stationary problem is solved numerically for values of contact angle close to a right angle. The existence of a film having the constant thickness is found. It is shown that this solution is stable when the gravity is low. Using numerical continuation method, value of the gravity for which this solution becomes unstable is found.
\end{abstract}

Keywords: liquid film, free surface, thin-layer approximation, thermocapillary effect, small disturbances, stability of solution.

DOI: 10.17516/1997-1397-2015-8-3-253-259

\section{Introduction}

This paper motivated to interpret results of experiments that were carried out by professors O. Kabov, L. Tadrist and there colleagues from Marseille [1]. This experiments result in free vertical liquid films that can be used in water desalination technology [2].

The fundamental tool to solve problems for liquid films is thin-layer approximation. The most part of papers on this subject considers films trickling down along the solid wall.

Thermocapillary effect plays a significant part in liquid motions [3-5].

In $[6,7]$ deformation of a free liquid film by thermocapillary forces is considered when the gravity is absent. In [6] temperature distribution for film is prescribed, meanwhile in [7] temperature is unknown function.

In [8] a deformation and a rupture of a thin liquid film which is hanging between two solid flat walls under the action of concentrated thermal load action are considered under micro-gravity conditions.

The paper [9] is devoted to the problem for infinite non-isothermal liquid film with thermoisolated free surfaces under gravitation. In the two-dimensional case when the thickness is constant, the exact solution of Navie - Stokes equations is found. A solution having the constant thickness is investigated for stability when wave number is small. Spectral problem for perturbations gives the solution in the form of damped oscillations.

*oksanabur@mail.ru

(c) Siberian Federal University. All rights reserved 


\section{Mathematical model}

Let the viscous incompressible thermo-conducting liquid occupy the infinite layer $\Omega_{t}=\left\{x_{1} \in\right.$ $\left.(-\infty, \infty) ; x_{2} \in(0, l) ; x_{3} \in\left(-h\left(x_{1}, x_{2}, t\right), h\left(x_{1}, x_{2}, t\right)\right)\right\}$, when acceleration of gravity $\mathbf{g}=(0-g 0)$ is opposed to $O x_{2}$ axis, $x_{3}= \pm h\left(x_{1}, x_{2}, t\right)$ are unknown free boundaries. When $x_{2}=0$ and $x_{2}=l$, the liquid is bounded by solid walls.

We assume that $\max h=\varepsilon l,|\nabla h|=O(\varepsilon), l \Delta h=O(\varepsilon)$ when $\varepsilon \rightarrow 0$, where $\nabla$ and $\Delta$ are the two-dimension gradient and the Laplacian. The longitudinal scale of the problem $l$ is much larger than the transversal scale $\varepsilon l$, i.e $\varepsilon<<1$.

Let us suppose that liquid density $\rho$, kinematic coefficient of viscosity $\nu$ and thermodiffusion coefficient $\chi$ are constant while the surface tension coefficient $\sigma$ is a linear function of temperature $T$ :

$$
\sigma=\sigma_{0}-\kappa\left(T-T_{0}\right)
$$

where $\sigma_{0}, \kappa$ and $T_{0}$ are positive constants. Let us denote by $\delta T$ the characteristic temperature difference and let suppose that $\kappa \delta T / \sigma_{0}=O\left(\varepsilon^{2}\right)$ when $\varepsilon \rightarrow 0$. We will assume the flow to be symmetric with respect to the plane $x_{3}=0$.

It is known ([10]), that for isothermal flow, stable film's length is no more than capillary radius. So now let the temperature is not constant.

Reasoning by analogy with [6] we obtain two equations connecting the flow rate vector $\mathbf{q}$ through the cross-section of the layer, the film thickness $h$ and the mean value $T^{*}\left(x_{1}, x_{2}, t\right)$ of its temperature using thin-layer approximation:

$$
\begin{aligned}
h_{t t}+\frac{\sigma_{0}}{\rho} \nabla \cdot(h \nabla \Delta h) & =\frac{\kappa}{\rho} \Delta T^{*}-\mathbf{g} \nabla h, \\
h_{t}+\nabla \cdot \mathbf{q} & =0 .
\end{aligned}
$$

It is follows from impermeability condition for solid walls that

$$
\mathbf{q}=0 \text { when } x_{2}=0, x_{2}=l .
$$

We obtain other pair of boundary conditions from prescribing three-phase contact angle:

$$
\frac{\partial h}{\partial x_{2}}=\alpha_{0} \text { when } x_{2}=0, \quad \frac{\partial h}{\partial x_{2}}=\alpha_{1} \text { when } x_{2}=l .
$$

We note that condition $\left|\alpha_{0}\right|,\left|\alpha_{1}\right|<<1$ must hold true in the framework of thin-layer approximation.

We assume that the free surfaces are thermo-isolated:

$$
\frac{\partial T}{\partial \mathbf{n}}=0 \text { when } x_{3}=h\left(x_{1}, x_{2}, t\right)
$$

where $\mathbf{n}$ is unit normal vector to the interfacial boundary.

The heat conduction equation can be written in the form:

$$
T_{t}+\left(\mathbf{v} \cdot \nabla_{3}\right) T=\chi \Delta_{3} T
$$

where $\mathbf{v}=\left(v_{1}, v_{2}, v_{3}\right)$ is velocity vector, $\nabla_{3}$ and $\Delta_{3}$ are the three-dimension gradient and the Laplacian. 
It follows from mean value theorem that there exist $\xi \in[0, h]$ and $\eta \in\left[x_{3}, \xi\right]$ such, that

$$
T\left(x_{1}, x_{2}, x_{3}, t\right)-T^{*}\left(x_{1}, x_{2}, t\right)=\frac{\partial T}{\partial x_{3}}\left(x_{1}, x_{2}, \eta, t\right) \cdot\left(x_{3}-\xi\right) .
$$

We note that $\left|x_{3}-\xi\right| \leqslant h \leqslant \varepsilon l$. So

$$
T=T^{*}+O(\varepsilon)
$$

Now we integrate left hand side of $(6)$ over the interval $[0, h]$ :

$$
\begin{aligned}
& \int_{0}^{h}\left(T_{t}+\left(\mathbf{v} \cdot \nabla_{3}\right) T\right) d z=h T_{t}^{*}+\mathbf{q} \nabla T^{*}+O(\varepsilon)=h T_{t}^{*}+\nabla \cdot\left(\mathbf{q} T^{*}\right)-T \nabla \cdot \mathbf{q}+O(\varepsilon)= \\
& =\left(h T^{*}\right)_{t}+\nabla \cdot\left(\mathbf{q} T^{*}\right)+O(\varepsilon),
\end{aligned}
$$

The last equality follows from (2).

Integration of the right hand side of (6) gives:

$$
\int_{0}^{h} \chi \Delta_{3} T d z=\chi h \Delta_{3} T^{*}+O(\varepsilon)
$$

It is follows from (5) that $\nabla h \cdot \nabla T^{*}=0$. So, $h \cdot \Delta T^{*}=\nabla \cdot\left(h \nabla T^{*}\right)$ and

$$
\int_{0}^{h} \chi \Delta_{3} T d z=\chi \nabla \cdot\left(h \nabla T^{*}\right)+O(\varepsilon) .
$$

The members of the order of $O(\varepsilon)$ can be neglected. We put $T\left(x_{1}, x_{2}, t\right):=T^{*}\left(x_{1}, x_{2}, t\right)$ and obtain the equation

$$
(h T)_{t}+\nabla \cdot(\mathbf{q} T)=\chi \nabla \cdot(h \nabla T) .
$$

The system of equations (1)-(2), (7) is the closed system connecting the flow rate, the film thickness and its temperature.

\section{Numerical solution of the one-dimensional stationary problem}

We consider the one-dimensional stationary problem. The system (1)-(2), (7) is written as

$$
\frac{\sigma_{0}}{\rho}\left(h^{\prime \prime \prime} h\right)^{\prime}=\frac{\kappa}{\rho} T^{\prime \prime}+g h^{\prime}, \quad\left(h T^{\prime}\right)^{\prime}=0, q=0 .
$$

We integrate the first two equations and eliminate $T$. Using (3) we obtain

$$
\frac{\sigma_{0}}{\rho}\left(h^{\prime \prime \prime} h\right)=\frac{\kappa b}{\rho h}+g h,
$$

where prime denotes differentiation in $x_{2}:=x, b=$ const.

We rewrite (8) in dimensionless form:

$$
h^{\prime \prime \prime} h=\frac{K}{\eta h}+\frac{h}{\eta}
$$

where $\frac{1}{\eta}=\frac{g l^{3} \rho}{\sigma_{0} \delta}, \frac{K}{\eta}=\frac{\kappa l^{3} b}{\sigma_{0} \delta^{3}}, \delta=\varepsilon \mathrm{MaCr}, \mathrm{Ma}=\kappa \delta T l / \rho \nu^{2}, \mathrm{Cr}=\kappa \delta T / \sigma_{0}$. 
We make the change $s=\frac{x}{\eta^{1 / 3}}$ of the independent variable for convenience in solving problem numerically. Equation (9) will be written as

$$
h \frac{d^{3} h}{d s^{3}}=\frac{K}{h}+h
$$

In addition to the boundary conditions (4) we specify condition

$$
h(0)=h_{0},
$$

that is equivalent to definition of the volume of the flow.

Note, that if $h_{0}=\sqrt{-K}$ and $\alpha_{0}=\alpha_{1}=0$ (the contact angle equals to $\pi / 2$ ), then the boundary value problem (10), (11), (4) has the constant solution $h=\sqrt{-K}$.

Now we will disturb the contact angle $\alpha_{1}$. The problem (10), (11), (4) is solved numerically using the shooting method. The numerical results are presented in Fig.1.

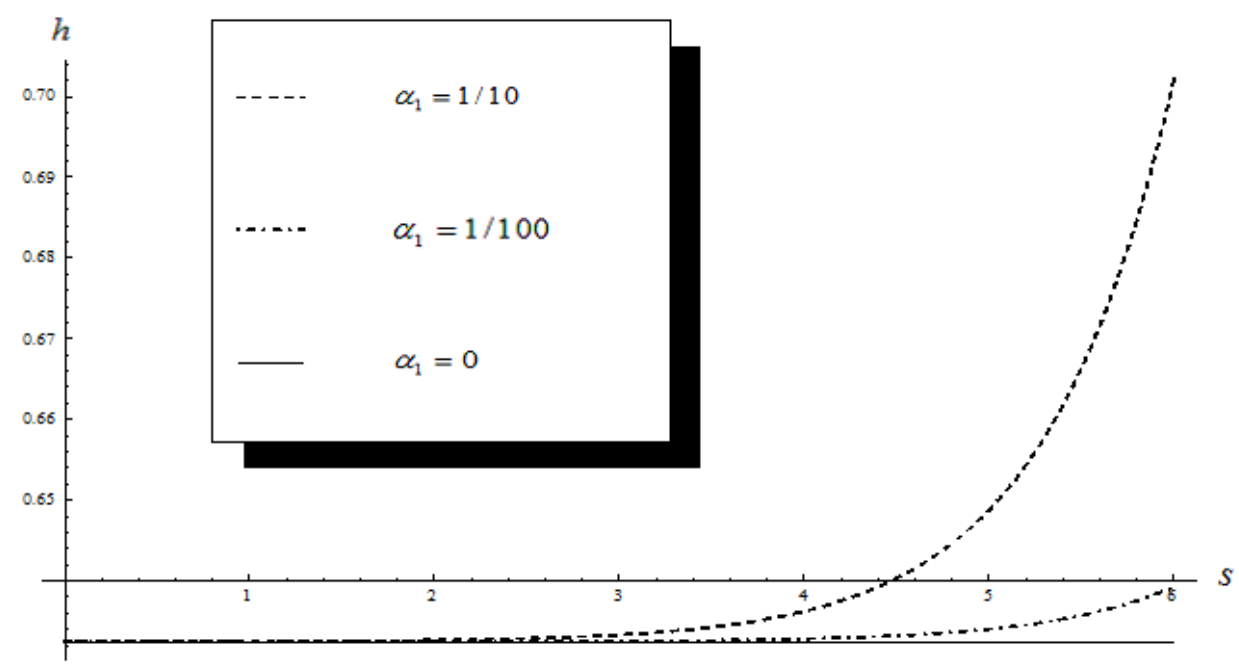

Fig. 1. The dependence of the thickness from the coordinate for various $\alpha_{1}$ when $\alpha_{0}=0$, $K=-2 / 5$

As can be seen from the calculation results, when the deviate of the contact angle from $\pi / 2$ is small, the solution is still exists, but when it grows, the solution begin to collapse.

\section{Stability of the solution}

As it is found, the system (1)-(2), (7) with boundary conditions (3)-(5) has the solution with a constant thickness. Let

$$
h_{0}=1 \text {, }
$$

Then

$$
q=0, T=b x+c,
$$


where $b=-\frac{\beta}{\gamma}, \beta=\mathrm{GaCr} /(\varepsilon \mathrm{Ma}), \gamma=\lim _{\varepsilon \rightarrow 0} \varepsilon^{-2} \mathrm{Cr}, \mathrm{Ga}=g l^{3} / \nu^{2}, c$ is a constant which means a characteristic value of the temperature.

We linearize the problem (1)-(2), (7), (3)-(5), closely to the solution (12)-(13) and obtain the system of equations for disturbances:

$$
\delta h_{t t}+h_{x x x x}=\gamma T_{x x}+\beta h_{x}, \quad T_{t}+b q=\alpha\left(b h_{x}+T_{x x}\right), \quad h_{t}+q_{x}=0
$$

with boundary conditions

$$
h_{x}(0)=h_{x}(1)=0, \quad T_{x}(0)=T_{x}(1)=0, \quad q(0)=q(1)=0,
$$

where $\alpha=1 /(\varepsilon \operatorname{MaPr}), \operatorname{Pr}=\nu / \chi$.

This system (14)-(15) is reduced to the equation for $h$ :

$$
\left(\left(\frac{\partial^{2}}{\partial x^{2}}-\frac{\partial}{\alpha \partial t}\right)\left(\frac{\partial^{4}}{\partial x^{4}}+\delta \frac{\partial^{2}}{\partial t^{2}}\right)-2 \beta \frac{\partial^{3}}{\partial x^{3}}\right) h=0
$$

with boundary conditions

$$
h_{x x x}-\beta h=0, \quad h_{x}=0, \quad \alpha h_{x x x x x}=\beta\left(2 \alpha h_{x x}+h_{t}\right) \text { when } x=0, x=1
$$

We seek the solution of the problem (16)-(17) in the form

$$
h(x, t)=e^{\lambda t} f(x)
$$

and obtain the spectral problem

$$
\begin{gathered}
\left(\left(\frac{\partial^{2}}{\partial x^{2}}-\frac{\lambda}{\alpha}\right)\left(\frac{\partial^{4}}{\partial x^{4}}+\delta \lambda^{2}\right)-2 \beta \frac{\partial^{3}}{\partial x^{3}}\right) f=0 \\
f_{x}(0)=f_{x}(1)=0, \quad f_{x x x}(0)-\beta f(0)=f_{x x x}(1)-\beta f(1)= \\
=f_{x x x x x x}(0)-\left(2 \beta f_{x x}(0)+\frac{\lambda}{\alpha} f(0)\right)=f_{x x x x x x}(1)-\left(2 \beta f_{x x}(1)+\frac{\lambda}{\alpha} f(1)\right)=0 .
\end{gathered}
$$

Assuming the parameter $\beta$ to be small, we expand the solution into series in the $\beta$ :

$$
f=f_{0}+\beta f_{1}+\ldots, \lambda=\lambda_{0}+\beta \lambda_{1}+\ldots
$$

From the boundary value problem (19), (20) in the zero and the first order, we obtain

$$
\begin{gathered}
\left(\lambda_{0}\right)_{n}=i \frac{n^{2} \pi^{2}}{\sqrt{\delta}}, \quad n \in Z \\
\lambda_{1}=0 .
\end{gathered}
$$

In the second order for $\alpha=0.002, \delta=0.01$, it is obtained that

$$
\lambda_{2}=-0.0000202508-0.000000267649 i
$$

for the first mode $(n=1)$. It means that the solution with a constant thickness is stable in the second approximation.

Then we make the parameter continuation for $\beta$ using orthogonalization method described in $[11,12]$. The values of $\lambda$ obtained are presented in Tab. 1 .

That is for $g<0,282406 \mathrm{~m} / \mathrm{s}^{2}$ the solution is stable and for $g>0,282406 \mathrm{~m} / \mathrm{s}^{2}$ it becomes unstable. 
Table 1. The values of $\lambda$ obtained using orthogonalization method for various $\beta$

\begin{tabular}{|l|l|l|}
\hline$g, m / s^{2}$ & $\beta$ & $\lambda$ \\
\hline 0 & 0 & $i \cdot 42,9075872459633$ \\
\hline 0,01 & 1,33 & $-0,000021+i \cdot 42,9083737144401$ \\
\hline 0,2 & 26,66 & $-0,000009146+i \cdot 42,9079533490524$ \\
\hline 0,282406 & 37,6541 & $i \cdot 42,9072745305347$ \\
\hline 0,4 & 53,33 & $0,0000072862+i \cdot 42,9073447541626$ \\
\hline 1,0 & 133,33 & $0,00004554646+i \cdot 42,9060715500626$ \\
\hline 4,0 & 533,33 & $0,00163450436266563+i \cdot 95,8899780393908$ \\
\hline 7,0 & 933,33 & $0,00380961170250427+i \cdot 72,4009517411667$ \\
\hline 9,8 & 1306,66 & $0,00637246347482759+i \cdot 61,0867150009782$ \\
\hline
\end{tabular}

\section{Conclusion}

Deformation of the free vertical liquid film by combined effect of gravity and thermocapillary forces is considered in the framework of thin-layer approximation. The system of equations connecting the flow rate, the film thickness and its temperature is obtained.

The solution $h=$ const, $T=b x, q=0$ of this system is found. Using the shooting method, one-dimensional stationary problem is solved numerically for values of contact angle close to $\pi / 2$.

Parameter $\beta$ is proportional to the gravitation. When $\beta$ is small, it is obtained, that the solution is stable in the second approximation. Using numerical continuation method, value of the gravity for which this solution became unstable is found.

Then it is planned to study the stability of the free liquid film taking into account the evaporation effect.

The author would like to thank V.V. Pukhnachov and V.V. Kuznetsov for helpful advices. This work is supported by the Russian Foundation for Basic Research (project 13-01-00526).

\section{References}

[1] W.Soua, A.Kaiss, L.Tadrist, O.Kabov, Hydrodynamic and Heat Transfer of a falling liquid film on a horizontal heated tube: Simulation and Experimentation, Third International Topical Team Workshop on two-phase systems for ground and space applications, Brussels, Belgium, September 10-12, 2008.

[2] H.Fridhi, W.Soua, A.Kaiss, L.Tadrist, Flow patterns and wavelength measurement for liquid film falling around horizontal tube, Conference: Composite Materials \& Renewable Energy Applications (ICCMREA), 2014 International Conference, 1-6.

[3] V.V.Pukhnachov, Thermocapillary convection under law gravity, Fluid Dynamics Transaction, 14(1989), 145-204.

[4] L.G.Napolitano, Thermodynamics and Dynamics of Surface Phases, Acta Astronaut, 5(1979), no. 5-6, 1093-1112. 
[5] V.A.Batishchev, V.V.Kuznetsov, V.V.Pukhnachov, Marangoni boundary layers, Prog. Aerospace Sci., 26(1989), 353-370.

[6] V.V.Pukhnachov, S.B.Dubinkina, Model of deformation and breakdown of a free nonisothermal film by thermocapillary forces, Trudy Ross. Akad. Nauk. Mekhanika Zhidkosti i Gaza, 2006, no. 5, 89-107 (in Russian).

[7] S.V.Meleshko, V.V.Pukhnachev, T.P.Pukhnacheva, Traveling waves and self-similar solutions in model of free non-isothermal liquid film, Advances in Mathematical Sciences and Applications, 14(2004), no. 5-6, 25-40.

[8] A.S.Ovcharova, Features of the rupture of free hanging liquid film under the action of a thermal load, Physics of Fluids, 23 (2011), no. 10, 102-106.

[9] O.A.Burmistrova, Stability of a vertical liquid film with consideration of the Marangoni effect and heat exchange with the environment, Journal of Applied Mechanics and Technical Physics, 55 (2014), no. 3, 386-393.

[10] A.D.Myshkis, V.G.Babskii, N.D.Kopachevskii, L.A.Slobozhanin, A.D.Tiuptsov, Hydromechanics of weightlessness, Moscow, Nauka, 1976 (in Russian).

[11] S.K.Godunov, Numerical Solution of Boundary-Value Problems for Systems of Linear Ordinary Differential Equations, Usp. Mat. Nauk, 16 (1961), no. 3, 171-174 (in Russian).

[12] A.A. Abramov, On the transfer of boundary conditions for systems of ordinary linear differential equations (a variant of the dispersive method), Zh. Vychisl. Mat. i Mat. Fiz, 1(1961), no. 3, 542-545 (in Russian).

\section{Равновесие и устойчивость свободной жидкой пленки в продольном поле тяжести}

\section{Оксана А. Бурмистрова}

\footnotetext{
Исследуется деформачия свободной вертикальной жидкой пленки при совместном действии сильи тяжести и термокапиллярных сил. В приближении тонкого слоя получена система уравнений, связывающая расход жидкости, толщину плёнки и её температуру. Методом стрелъбъ одномерная стационарная задача численно решена при значениях краевого угла, близких к прямому. Обнаружено существование плёнки с постоянной толщиной. Для этого решения показана устойчивость при пониженной гравитации. С помощъю метода продолжения по параметру найдено значение ускорения тяжести, при котором решение становится неустойчивым.
}

Ключевые слова: жидкая плёнка, свободная поверхность, приближение тонкого слоя, термокапиллярный эффект, малые возмущения, устойчивость решения. 